Research Article

\title{
Research on a Dynamic Evaluation of Financial Risks of Intellectual Property Pledge Financing of High-Tech Enterprises
}

\author{
Xiangyu Ge $\mathbb{D}$, ${ }^{1}$ Ying Jin, ${ }^{1,2}$ Qing Li $\mathbb{D}^{1},{ }^{1}$ Xiaofang Yao, ${ }^{1}$ and Shican Liu $\mathbb{D}^{1}$ \\ ${ }^{1}$ School of Statistics and Mathematics, Zhongnan University of Economics and Law, Wuhan 430 073, China \\ ${ }^{2}$ School of Statistics and Big Data, Henan University of Economics and Law, Zhengzhou 450 046, China \\ Correspondence should be addressed to Shican Liu; canbulajiji@163.com
}

Received 17 October 2021; Accepted 2 December 2021; Published 26 December 2021

Academic Editor: Dehua Shen

Copyright (c) 2021 Xiangyu Ge et al. This is an open access article distributed under the Creative Commons Attribution License, which permits unrestricted use, distribution, and reproduction in any medium, provided the original work is properly cited.

Intellectual property pledge financing is effective in alleviating the financing problems of scientific and technological enterprises to a certain extent. However, compared with traditional loans, intellectual property pledge financing is at greater risk with an eye to the particularity of intellectual property pledges. Therefore, it is very important to evaluate the risk of intellectual property pledge financing. This article first outlines the information entropy method, functionalizing indicator data and weight data, and applies the combination of the weight function and indicator function in dynamic evaluation function, to get the final evaluation result. Second, taking four high-tech listed companies on the growth enterprises market (GEM) as example, an evaluation indicator system has been constructed with 16 relevant financial indicators from 2015 to 2019. Then, based on the dynamic function method, we can construct the evaluation function to describe the change trend of the financial indicators of enterprises with a comparison of the advantages and disadvantages of the financial indicators of enterprises. Finally, this article has made a comprehensively comparative analysis on the dynamic evaluation, and the financial risk of intellectual property pledge financing of high-tech enterprises has been done with the combination of the dynamic evaluation curve with the optimal time weight determined by the maximum entropy method.

\section{Introduction}

The key of economic and social development to promoting high-quality development is innovation, particularly scientific and technological innovation. Therefore, it is encouraged to carry out scientific and technological innovation for enterprises to implement the strategy of innovationdriven development, which is the main task of economic and social development in China. Nowadays, high-tech enterprises have become the backbone of economic development and innovative growth and play an important role in gathering scientific and technological resources, creating intellectual property rights, and driving economic transformation. The core competency of high-tech enterprises is to accelerate the transformation rate of scientific and technological research. However, most high-tech enterprises are new on a small scale, which are not available with appropriate fixed assets as collateral and fail to get funds from banks and other financial institutions, facing the predicament of lacking funds. Financing difficulties limit the development of most high-tech enterprises to some extent.

According to the data released by the Chinese State Intellectual Property Office from 2007 to 2020, the number of valid invention patents in China (excluding Hong Kong, Macao, and Taiwan) has increased from 84,000 to 2.213 million, with the invention of patents per 10,000 population reaching 15.8 . With the significantly increasing ownership of China's intellectual property, it is evident that China is a real intellectual property power. Now, many high-tech enterprises in China are fitted with advanced patent technologies, which are valuable in the market. At the same time, financing a loan of intellectual property pledge effectively offers a loan to solve the financing difficulties to promote the technical innovation of these small- and medium-sized hightech enterprises with advanced patents. As for the current state and government departments, some relevant policies 
have been issued by governments to promote the development of local intellectual property pledge financing businesses. Unfortunately, in reality, intellectual property pledge financing has a long way to go. The key point is to evaluate and control the financial risk of high-tech financing enterprises. Therefore, the assessment and monitoring of financial risk of the enterprise are obvious to be particularly important, which has always been one of the main reasons affecting the development of intellectual property pledge financing business. It is of great practical significance to discuss the dynamic evaluation of financial risk in intellectual property pledge financing of high-tech enterprises.

In recent years, attention has been paid to the financing of small and medium enterprises (SMEs) in the field of finance. Significant analysis has been presented by Banerjee et al. [1], who believed that small and medium financial institutions have advantages in terms of information and cost to provide services over SMEs. Angelini et al. [2] found that relational lending featured with long term and concentration of trading objects are supposed to improve the availability and terms of loans for SMEs. The influence of government policies and national financial structure on the credit availability of SMEs has been reported by Berger and Udell [3]. Another research on a new financing model for SMEs based on guarantees has been studied by Zhang and Zhang [4]. Most researches have analyzed the relationship between bank credit and SMEs financing (see Lin and Li [5] and $\mathrm{Xu}$ and $\mathrm{Zou}[6]$ ), while the interest rate and guarantee of SMEs loans have been discussed by Xiong et al. [7, 8]. Meanwhile, intellectual property pledge financing has been studied by many scholars in many aspects. The research of Jarboe and Ellis [9] focused on the innovative financing of intangible assets in the United States, including improvement of the value evaluation, trading mechanism of intellectual property, characteristics of intellectual property, and the disclosure of intellectual property information with policy suggestions proposed. Crawford and Strasser [10] proposed a series of measures to punish intellectual property infringement. However, Chessy and Toivanen [11] found that financing risk is not significantly correlated with the value of pledges. The study by Niinimaki [12] also believed that risk types cannot be identified simply by the value of objects. The financing behavior of intellectual property pledges and their legal uncertainty has been reported by Davies [13]. Detailed information of the risks in different stages of intellectual property has been studied from formation to pledge by Pennington and Sanchez [14].

The emphasis of existing literature is mainly placed on the risk sources and formation mechanism of intellectual property pledges. As the subject of the intellectual property pledge loan is still the enterprise itself, the enterprise's operation income is regarded as the first repayment source of the intellectual property pledge loan. When financial problems happen to a pledge financing enterprise happens, there will definitely be a risk of default. If a pledge financing enterprise is allowed to dispose of intellectual property rights, the risk is greater than the mortgage of tangible assets. Therefore, it is necessary to comprehensively evaluate the financial situation of pledge financing enterprises. It is more likely to minimize the cost of default risk when the problem is found early; the sooner, the better. The method commonly used in science and technology evaluation consists of the AHP analysis method by Schenkerman [15], fuzzy comprehensive evaluation method by Chen and Hwang [16], DEA evaluation method by Ding et al. [17], and VIKOR method by Liu et al. [18].

In the era of big data, traditional data analysis technology is faced with many limitations in information data mining. Functional data analysis is a discrete realization regarding observation data as a random process to analyze the changing pattern of data from the perspective of the whole function, which not only relax the structural constraints and distribution assumptions of data collection but also dig down inside the deep dynamic change patterns of data. Overall, it is of great significance to enrich information mining technology in the era of big data. The implementation of the traditional dynamic comprehensive evaluation method is mainly based on the cross-sectional data and time series data, which turns out to be discrete data in the process of evaluation, including original data, weight, and final evaluation. With the fusion of the cross-sectional data and the time series data, the original data have been changed to the functional data, the concept of which has been proposed by Ramsay [19], formed by the function feature of time as the independent variable. Later, the improvement of the analysis framework system has been further studied by several world-renowned statisticians such as Ramsay and Dalzell [20], Silverman [21], Lu [22], Delagle and Hall [23], Petersen and Muller [24], and Kokoszka and Reimherr [25] with the result of relaxing the structural constraints and distribution setting of data collection and deeply digging the potential dynamic information of data from the perspective of the interactive derivative function. Many successful explorations have been made on the expansion of classical statistical methods in functional data, for example, the principal component analysis [21], discriminant analysis [26], linear model [27], principal differential analysis [28], panel data model [29], and cluster analysis [30]. Based on the process of functional data, it is easy to carry out the dynamic comprehensive evaluation function curve for a period of time, which is more useful and effective for evaluation analysis.

In previous literature, the comprehensive evaluation of the risk of intellectual property pledge financing focuses on static analysis [31, 32]. Meanwhile, the comprehensive evaluation index for the small commodity market has been constructed by the functional data analysis, with making the dynamic evaluation of the quality of life $[33,34]$. This is the first time to evaluate the financial risk of intellectual property pledge financing enterprises by functional data analysis in the article.

In this article, the function analysis method has been used to evaluate the financial risk of intellectual property pledge financing enterprises. Compared with the risk evaluation with annual data, quarter data with shorter time intervals are better to reflect the function characteristics of data in the case of time as an independent variable, which is more accurate and effective in describing the financial status 
of enterprises in time. Whether at a specific moment or the overall time interval, the objectivity and comparability of the evaluation results are obvious.

The rest of the article is organized as follows. In Section 2, we will establish theoretical models and fractionalize the indicator data and the weight data to obtain the dynamic evaluation function with a combination of the weight function and the indicator function. In Section 3, the system of intellectual property pledge financing risk evaluation indicator will be constructed. In Section 4, we will make a dynamic evaluation with the functional curve for four high-tech listed enterprises, with a comprehensive comparison of the intellectual property pledge financing risk. A conclusion of this article is given in Section 5.

\section{The Risks Evaluation Method of Intellectual Property Pledge Financing}

Intellectual property pledge financing is indeed risky with great uncertainty. If the default happens to enterprises, debt assets are difficult to realize with creating a chain reaction, which requires financial institutions to evaluate the financial status of enterprises' pledge financing. Based on the quarterly financial data to be evaluated, the process of the generation of sample data is treated as a functional process. Regarding the observed data as a whole, the functional data analysis method is used to fit the overall function process of the observed discrete data to get the overall curve of financial data and reflect the dynamic changes of financial data. According to the weight function, the comprehensive evaluation value of finance can be obtained to judge the financial risk of enterprises. The specific theoretical model is as follows.

2.1. Functional Data Analysis Methods. The basic idea of functional data analysis (FDA) is to treat each sample observation as a whole rather than a sequence of individual observations. Due to errors or noise in the original data, the relationship between the actual observed data and the underlying function is as follows:

$$
y_{h}=x\left(t_{h}\right)+\varepsilon_{h}, \quad h=1,2, \ldots, T,
$$

where $\varepsilon_{h}$ represents the "roughness" of the original data due to disturbances, errors, deviations, and other exogenous factors. The essence of transforming observed discrete data into functional data is to recover the underlying data from the original data. It is assumed that generating data are a smooth process, and the process of transforming the original discrete data into smooth function data has been realized based on the linear combination of basis functions, including Fourier basis functions, $B$-spine basis functions, polynomial basis functions, and wavelet basis functions, in which the Fourier base function and $B$-spine base function are more widespread. For periodic data, the Fourier base function is suitable. However, for aperiodic data, the $B$-spine base function is suitable. For a set of basis functions $\phi_{k}$, $k=1,2, \ldots, K$, estimation of $x(t)$ can be obtained by the linear combination of the basis functions as follows:

$$
\widehat{x}\left(t_{h}\right)=\sum_{k=1}^{K} c_{k} \phi_{k}\left(t_{h}\right) .
$$

It is known that the choice of basis function is also important to estimate the following derivative:

$$
D^{m} \widehat{x}\left(t_{h}\right)=\sum_{k=1}^{K} c_{k} D^{m} \phi_{k}\left(t_{h}\right)
$$

where $D^{m} \widehat{x}\left(t_{h}\right)$ means the $m$-order derivative of the function, usually $m=2$. Related to the choice of a basis function, the estimation of the function is required to be considered, along with the estimation of the first derivative and the second derivative.

When a linear combination of basis functions is used to smooth the data, the coefficients $c_{k}, k=1,2, \ldots, K$, are needed to determine, by the least-squares criterion, i.e.,

$$
\min \sum_{h=1}^{T}\left[x\left(t_{h}\right)-\widehat{x}\left(t_{h}\right)\right]^{2},
$$

or

$$
\min \sum_{h=1}^{T}\left[x\left(t_{h}\right)-\sum_{k=1}^{K} c_{k} \phi_{k}\left(t_{h}\right)\right]^{2} .
$$

Assuming that the value of the basis function $\phi_{k}\left(t_{h}\right)$ $(k=1,2, \ldots, K)$ at the observation point $t_{h}(h=1,2, \ldots, n)$ with $n \times K$-order matrix as $\Phi=\phi_{k}\left(t_{h}\right)$, where $\Phi$ is full rank, then the minimized solution is as follows:

$$
c=\left(\Phi^{\prime} \Phi\right)^{-1} \Phi^{\prime} x .
$$

However, the control of the expansion is not flexible enough in the degree of uniform smoothness without continuous control ability. Therefore, in the practical analysis, the main concern is the degree of fitting and smoothness of the fitting function. In general, the higher the degree of the fitting is, the greater the volatility of the fitting function is. To ensure the smoothness of the fitting, a penalty term is introduced in the object function:

$$
\begin{aligned}
\text { PENSSE } & =\min \sum_{h=1}^{T}\left[x\left(t_{h}\right)-\widehat{x}\left(t_{h}\right)\right]^{2}+\lambda \int_{T}\left\{D^{2} x(t)\right\} \mathrm{d} t \\
& =\min \sum_{h=1}^{T}\left[x\left(t_{h}\right)-\sum_{k=1}^{K} c_{k} \phi_{k}\left(t_{h}\right)\right]^{2}+\lambda \int_{T}\left\{D^{2} x(t)\right\} \mathrm{d} t .
\end{aligned}
$$

The first term is the sum of squares of residuals, which indicates the degree of fitting; and the penalty term is added to indicate the degree of fluctuation. Specifically, $\lambda$ means a measure between the degree that the function of $x(t)$ fits the data and the volatility of the function itself, which is called a smoothing parameter. When $\lambda \longrightarrow 0$, the smooth fitting curve $x\left(t_{h}\right)$ is the interpolation function of the data, with no arbitrary fluctuation interpolation curves, which are the smoothest second-order differentiable function of the data curve accurately fitted. When $\lambda \longrightarrow \infty, \int_{T}\left\{D^{2} x(t)\right\} \mathrm{d} t$, the 
smooth fitting curve of $x\left(t_{h}\right)$ is the standard linear regression of the data. More attention has been paid to the smoothness of the function as $\lambda$ gets bigger, with an estimated value as follows:

$$
c=\left(\Phi^{\prime} \Phi+\lambda \int_{T}\left\{D^{2} x(s)\right\}\left\{D^{2} x^{\prime}(s)\right\} \mathrm{d} s\right)^{-1} \Phi^{\prime} x .
$$

By comparing equations (6) and (8), it can be found that they are equal when $\lambda=0$. The data smoothing degree can be varied with the change of $\lambda$, a vector including the elements of node number of $B$-spline base. It is easy to estimate $\lambda$ using the generalized cross-validation (GCV) method.

\subsection{Comprehensive Evaluation Method of Pledge Financing} Risks. In this subsection, we will first introduce the dimensionless processing method of the data, followed by using the information entropy method to obtain the objective weight of the indicators and functionalizing the indicator data and the weight data to obtain a function curve of the comprehensive evaluation, resulting in determining the weight of the time measurement by the maximum entropy method. Combining the evaluation function to obtain the final evaluation result is expected. Before we go into the details, we have to introduce some notations. $S=\left\{s_{1}\right.$, $\left.s_{2}, \ldots, s_{n}\right\}$ means the number of evaluated objects to $n, X=$ $\left\{x_{1}, x_{2}, \ldots, x_{m}\right\}$ shows the number of evaluation indicators to $m$, and $H=\left\{t_{1}, t_{2}, \ldots, t_{T}\right\}$ are the time of $T . x_{i j}\left(t_{h}\right)$ represents the observed value of indicator $x_{j}(j=1,2, \ldots m)$ of the evaluated object $s_{i}(i=1,2, \ldots n)$ at time $t_{h}$ $(h=1,2, \ldots T)$.

2.2.1. Dimensionless Processing of Data. For an interval-type indicator, the optimal value is in a certain interval, which is transformed into a positive indicator:

$$
x_{i j}^{*}\left(t_{h}\right)= \begin{cases}1-\frac{a-x_{i j}\left(t_{h}\right)}{M}, & x_{i j}\left(t_{h}\right)<a, \\ 1, & a \leq x_{i j}\left(t_{h}\right) \leq b, \\ 1-\frac{x_{i j}\left(t_{h}\right)-b}{M}, & x_{i j}\left(t_{h}\right)>b,\end{cases}
$$

where $M=\max \left\{a-\min \left\{x_{i j}\left(t_{h}\right)\right\}, \max \left\{x_{i j}\left(t_{h}\right)\right\}-b\right\}$.

For dimensionless data, the complete process of sequence extremum processing is conducted using the following formulas reducing the dimension, which formula the forward type indicator/backward type indicator. Forward type indicator is shown as follows:

$$
x_{i j}^{*}\left(t_{k}\right)=\frac{x_{i j}\left(t_{k}\right)-\min _{i, k}\left\{x_{i j}\left(t_{k}\right)\right\}}{\max _{i, k}\left\{x_{i j}\left(t_{k}\right)\right\}-\min _{i, k}\left\{x_{i j}\left(t_{k}\right)\right\}}+1 .
$$

Backward type indicator is shown as follows:

$$
x_{i j}^{*}\left(t_{h}\right)=\frac{\min _{i, h}\left\{x_{i j}\left(t_{h}\right)\right\}-x_{i j}\left(t_{h}\right)}{\max _{i, h}\left\{x_{i j}\left(t_{h}\right)\right\}-\min _{i, h}\left\{x_{i j}\left(t_{h}\right)\right\}}+1 .
$$

2.2.2. Information Entropy Method for Calculating Objective Weight of Indicators. The entropy method is to determine the weight of an indicator by comparing the amount of information of the indicator. The concept of entropy is introduced from thermodynamics. In information theory, entropy is used to measure the degree of uncertainty, also known as the average amount of information. If the amount of information is more, the understanding of the objective function is accordingly more comprehensive. However, if the uncertainty is smaller, the impact on the system is smaller, and the corresponding target entropy is smaller. The reverse is also true.

The information entropy method of calculating the objective weight of indicators contains the following four steps:

Firstly, the characteristic proportion of each indicator is calculated. The characteristic proportion of the evaluated object of $i$ under indicator of $j$ in year of $t_{k}$ is as follows:

$$
p_{i j}\left(t_{h}\right)=\frac{x_{i j}^{*}\left(t_{h}\right)}{\sum_{i=1}^{n} x_{i j}^{*}\left(t_{h}\right)},
$$

where $x_{i j}^{*}\left(t_{h}\right)>0$ and $\sum_{i=1}^{n} x_{i j}^{*}\left(t_{h}\right)>0$.

Secondly, the entropy of each indicator is calculated. The entropy value of indicator $j$ in year of $t_{h}$ is as follows:

$$
e_{j}=-\frac{1}{\ln m} \sum_{i=1}^{n} p_{i j}\left(t_{h}\right) \ln p_{i j}\left(t_{h}\right) \text {. }
$$

Next, we calculate the difference coefficient ratio of indicators:

$$
g_{j}=1-e_{j}, \quad j=1,2, \ldots, m .
$$

Lastly, we determine the weight coefficient based on the difference coefficient:

$$
w_{j}=\frac{g_{j}}{\sum_{j=1}^{m} g_{j}}, \quad j=1,2, \ldots, m
$$

2.2.3. Function Curve of Comprehensive Evaluation. The basis function method is used to express the data as smooth curves. Generally speaking, the estimation of $\lambda$ is obtained by the GCV method.

$$
\operatorname{GCV}(\lambda)=\frac{\text { SSE }}{\left[\operatorname{trace}\left(I-S_{\phi, \lambda}\right)\right]^{2}},
$$

where

$$
\begin{aligned}
\text { SSE } & =\int_{T} \sum_{i=1}^{n} \sum_{j=1}^{m}\left(y_{i j}(t)-x_{i j}(t)\right)^{2} \mathrm{~d} t, \\
S_{\phi, \lambda} & =\Phi M(\lambda)^{-1} \Phi^{T} W, \\
M(\lambda)^{-1} & =\Phi^{T} W \Phi+\lambda R, \\
R & =\int_{T}\left\{D^{2} \Phi(t)\right\}\left\{D^{2} \Phi^{\prime}(t)\right\} \mathrm{d} t .
\end{aligned}
$$


After transforming the discrete data into functional data, the fitting curve $x_{i j}(t)$ of $m$ indicator data of each evaluated object and the fitting curve $w_{i j}(t)(j=1,2, \ldots, m)$ of weight function are found. The evaluation function curve is described by substituting the fitting curve into the evaluation model (18).

$$
y_{i}(t)=w_{1}(t) x_{i 1}(t)+w_{2}(t) x_{i 2}(t)+\cdots+w_{m}(t) x_{i m}(t) .
$$

2.2.4. Determining the Weight of Time Measurement. During the observation period, the influence of different time data on the total evaluation function is often different. Therefore, the weight of time measurement needs to be considered. Compared to the present impact, the past impact is relatively small. Thereupon, the evaluation of an observation object can be estimated by the following formula:

$$
\bar{y}_{i}=\frac{1}{T} \int_{T} w_{a}(t) y_{i} \mathrm{~d} t, \quad i=1,2, \ldots, n,
$$

where $w_{a}(t)$ denotes the time weight function. In particular, if it is considered to have the same influence on any time, the time weight function of $w_{a}(t)$ represents a straight line parallel to the $X$-axis. In this case, equation (19) can be abbreviated as follows:

$$
\bar{y}_{i}=\frac{1}{T} \int_{T} y_{i}(t) \mathrm{d} t
$$

Among them, the time weight is determined using the maximum entropy nonlinear programming method, which reveals the sample information as much as possible and takes into account the difference in the time sequence of the evaluated objects.

If the time weight vector is designed as $W_{a}=\left(W_{a 1}, W_{a 2}, \ldots, W_{a T}\right)^{T}$, the time degree can be taken into consideration as follows [35]:

$$
\alpha=\sum_{h=1}^{T} \frac{T-k}{T-1} w_{a h},
$$

where $\alpha$ reflects the importance degree of each period. When the value of $\alpha$ is closer to 0 , it means that the comprehensive evaluation pays more attention to the data at the recent period; when the value of $\alpha$ is closer to 1 , it means that the comprehensive evaluation pays more attention to the data at the earlier period; but when $\alpha=0.5$, it means that the comprehensive evaluation is paid equal attention to the data at each period. Therefore, the weight of time measurement can be solved by the following nonlinear programming method:

$$
\begin{aligned}
& \max \left\{-\sum_{h=1}^{T} w_{a h} \ln w_{a h}\right\}, \\
& \text { s.t. }\left\{\begin{array}{l}
\alpha=\sum_{h=1}^{T} \frac{T-k}{T-1} w_{a h}, \\
\sum_{h=1}^{T} w_{a h}=1,\left(w_{a h} \in[0,1]\right), h=1,2, \ldots, T .
\end{array}\right.
\end{aligned}
$$

In this way, a more objective and reasonable measurement of the weight of time can be obtained.

\section{Risks Evaluation Indicator System of Intellectual Property Pledge Financing}

As the enterprise has a role as the subject of intellectual property pledge loans, it is general to know that the first repayment source of intellectual property pledge financing is the cash flow earned from the enterprise's investment or other activities. The financial operation situation provides a comprehensive performance of enterprise development, which is the most direct embodiment of the current development situation and development potential of the enterprise. Moreover, the financial operation of the enterprise has a very important impact on both investors and other external stakeholders, as well as the development of the enterprise itself. When it comes to deterioration, not only will it affect the confidence and decision-making of external investors, but also invisible pressure will be put on the internal personnel of the enterprise and enthusiasm of research and development of scientific researchers, furthermore leading to the decrease of realization of intellectual property value. Therefore, it is a direct concern with the future development of the enterprise. The better the enterprise is, the more the funds will be invested in intellectual property. Even though the scale of the enterprises is limited, it is difficult for small- and medium-sized businesses to face more financial risks.

Based on previous research methods and the principles of scientifically, operability, independence, and comparability, 16 financial indicators have been selected from the six aspects, debt paying ability, profitability, operating ability, growth ability, cash flow indicators, and innovation ability, to construct an evaluation indicator system as shown in Table 1.

The debt paying ability is directly related to the survival of the enterprise, which is the basic premise of the healthy development of the enterprise. As a reflection of its continuous operating ability, the debt paying ability of an enterprise is applied to predict its future earnings and analyze its financial risks, which is measured by current ratio, assetliability ratio, and current liability ratio. 
TABLE 1: Risks evaluation indicator system.

\begin{tabular}{lc}
\hline First-level indicator & Second level indicator \\
\hline $\begin{array}{l}\text { Debt paying ability } \\
\text { Profit ability } \\
\text { Operating ability }\end{array}$ & $\begin{array}{c}\text { Current ratio, asset-liability ratio, current liability ratio } \\
\text { Operating profit ratio, return on total assets ratio, return on equity } \\
\text { Inventory turnover ratio, total assets turnover, fixed assets turnover }\end{array}$ \\
$\begin{array}{l}\text { Growth ability } \\
\text { Cash flow }\end{array}$ & $\begin{array}{c}\text { Net profit growth rate, total assets growth rate, } \\
\text { Increase rate of main business revenue }\end{array}$ \\
\hline Innovation ability & $\begin{array}{c}\text { Net cash flow from operating activities per share, } \\
\text { Cash ratio of operating income, ratio of cash to current liability }\end{array}$ \\
\hline
\end{tabular}

Profit ability refers to earning profits to ensure the continuous operation of the enterprise. Profit is regarded as the central concern for business operators, investors, and creditors. Improving profitability will strengthen the growth in the future and, in turn, will become more resilient to risks, which is represented by the income from patented technologies or products revenue, the characteristics of hightech enterprises. As a result, the profitability of enterprises is measured by operating profit rate, return on total assets ratio, and return on equity in the article.

The ability to make a profit using various assets is defined as the operating ability of enterprises, measured by inventory turnover ratio, total assets turnover, and fixed assets turnover to reflect the efficiency of economic resource management of high-tech enterprises in this article.

In order to better reflect the development potential of the enterprise in the future, the growth ability can be pictured by net profit growth rate, total assets growth rate, and increased rate of main business revenue.

Good enterprise cash flow is an important guarantee of enterprise financial stability and efficient operation, which can be illustrated by net cash flow from operating activities per share, cash ratio of operating income, and the ratio of cash to current liability.

The innovation ability of enterprises has some impacts on the financial risk of intellectual property pledge financing of high-tech enterprises. Due to the limitation of the quarterly data selection, the intangible asset ratio is provided to reflect the innovation ability.

\section{Dynamic Evaluation and Analysis of the Risks of Intellectual Property Pledge Financing}

Based on the time series data of the financial indicators of the financing enterprise, combined with the data related to the enterprise patent research and development and application, by the entropy method to make a dynamic comprehensive evaluation, obtaining the comprehensive weight of the distribution of the pros and cons of the evaluation data relative to the overall development level at different moments. Then, the comprehensive weighted dynamic evaluation method is used to better measure the dynamic risk of pledge financing enterprises. In this article, we consider high-tech listed companies as research objects, Wangsu Technology (Wangsu Keji), Shuzhi Technology (Shuzhi Keji), Hekang New Energy (Hekang Xinneng), Sanju
Environmental Protection (Sanju Huanbao), the quarter data from 2015 to 2019 of the CSMAR database, and some other data from the annual financial reports of listed enterprises.

The objective weight of risk evaluation indicators is shown in Table 2.

The data are fitted by adding penalties. Since the indicator of all samples is nonperiodic, the data can be represented as smooth curves by the basis function method. In general, the process of fitting is realized by the fourth-order spline basis with the same basic function and different smoothing parameters. When the smoothing parameter of $\lambda$ is large, the smoothness of the fitting curve is good. When it comes to being small, the volatility of the fitting curve is large. GCV is used to estimate the value of $\lambda$ with the lowest indicator GCV (as shown in Figure 1) to fit the original data to obtain the best fitting curve.

Moreover, the weight function is integrated to obtain the objective weight of each indicator. From Table 2, the weights of each first-level indicator are ranked as follows:

debt paying ability $>$ cash flow $>$ operating ability

$>$ innovation ability $>$ profit ability $>$ growth ability.

In Figure 2, even though there are some changes in the dynamic evaluation curve of Wangshu Keji from 2015 to 2019, the overall trend of the evaluation curve remains the same. On the other hand, the dynamic evaluation curve of Shuzhi Keji from 2015 to 2019 is a downward trend, with a short-term rebound. In particular, from 2018 to 2019, the evaluation curve of Shuzhi Keji is far lower than that of the other three enterprises. For Hekang Xinneng, there is a strong upward trend of the evaluation curve with the ups and downs from 2015 to 2019. For Sanju Huanbao, the dynamic evaluation curve from 2015 to 2019 has continued its steady upward trend, which is obviously higher than the other three curves. The maximum evaluation curve is in the second quarter of Wangshu Keji in 2015, whereas the minimum evaluation curve is in the first quarter of Shuzhi Keji in 2018.

As for Wangsu Keji, a rising trend in the evaluation curve is observed from the first quarter to the second quarter in 2015. In Figure 2, it has gradually slowed down to reach the peak value at the end of the second quarter. From the third quarter of 2015 to the third quarter of 2018, the evaluation curve has experienced an inverted V-shape, which reaches its bottom in the first quarter of 2017, and begins to rise. From Figures 3 and 4, great changes in the 
TABLE 2: Objective weight of risk evaluation indicators.

\begin{tabular}{|c|c|c|c|}
\hline First-level indicator & Second level indicator & Weight 1 & Weight 2 \\
\hline \multirow{3}{*}{ Debt paying ability } & Current ratio & 0.041 & \multirow{3}{*}{0.306} \\
\hline & Asset-liability ratio & 0.199 & \\
\hline & Current liability ratio & 0.066 & \\
\hline \multirow{3}{*}{ Profitability } & Operating profit ratio & 0044 & \multirow{3}{*}{0.107} \\
\hline & Return on total assets ratio & 0.032 & \\
\hline & Return on equity & 0.031 & \\
\hline \multirow{3}{*}{ Operating ability } & Inventory turnover ratio & 0.051 & \multirow{3}{*}{0.162} \\
\hline & Total assets turnover & 0.051 & \\
\hline & Fixed assets turnover & 0.06 & \\
\hline \multirow{3}{*}{ Growth ability } & Net profit growth rate & 0.01 & \multirow{3}{*}{0.103} \\
\hline & Total assets growth rate & 0.066 & \\
\hline & Increase rate of main business revenue & 0.027 & \\
\hline \multirow{3}{*}{ Cash flow } & Net cash flow from operating activities per share & 0.044 & \multirow{3}{*}{0.178} \\
\hline & Cash ratio of operating income & 0.076 & \\
\hline & Net cash flow from operating activities per share & 0.058 & \\
\hline Innovation ability & Intangible asset ratio & 0.144 & 0.144 \\
\hline
\end{tabular}

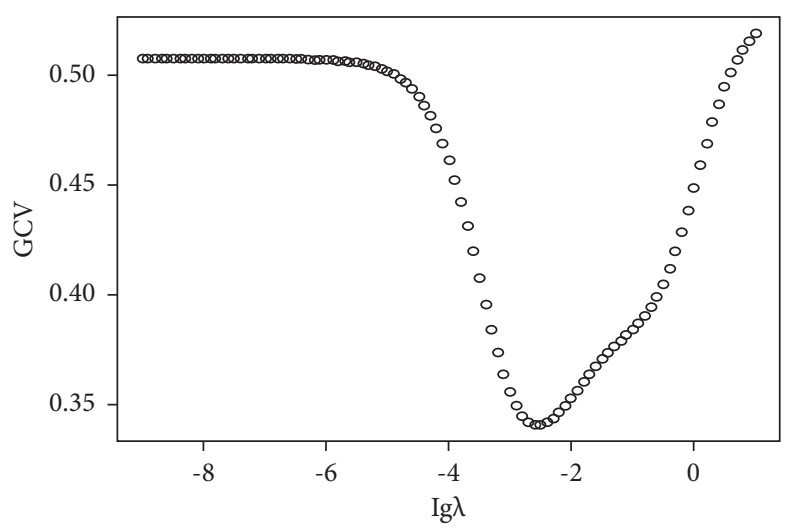

FIgURE 1: GCV curve.

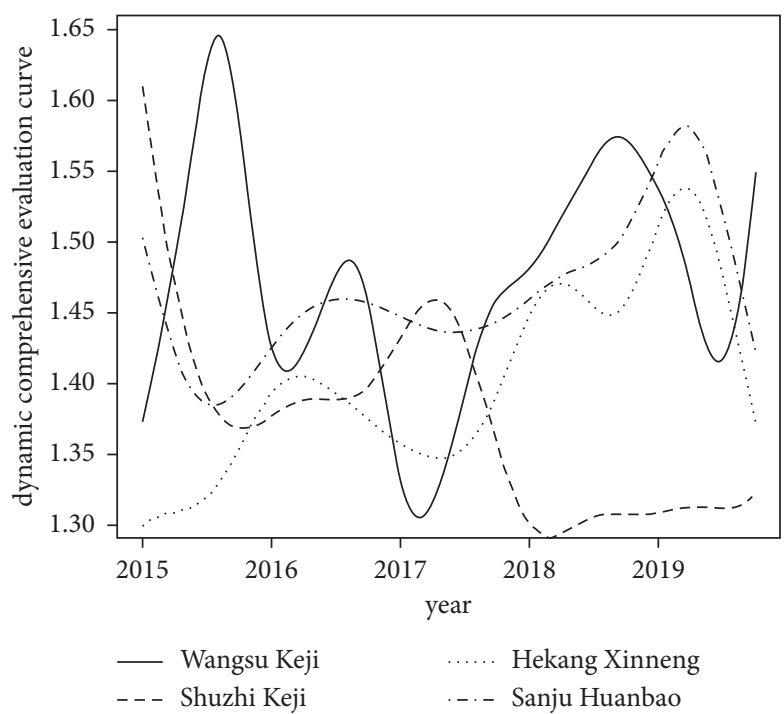

Figure 2: Dynamic comprehensive evaluation curve. 


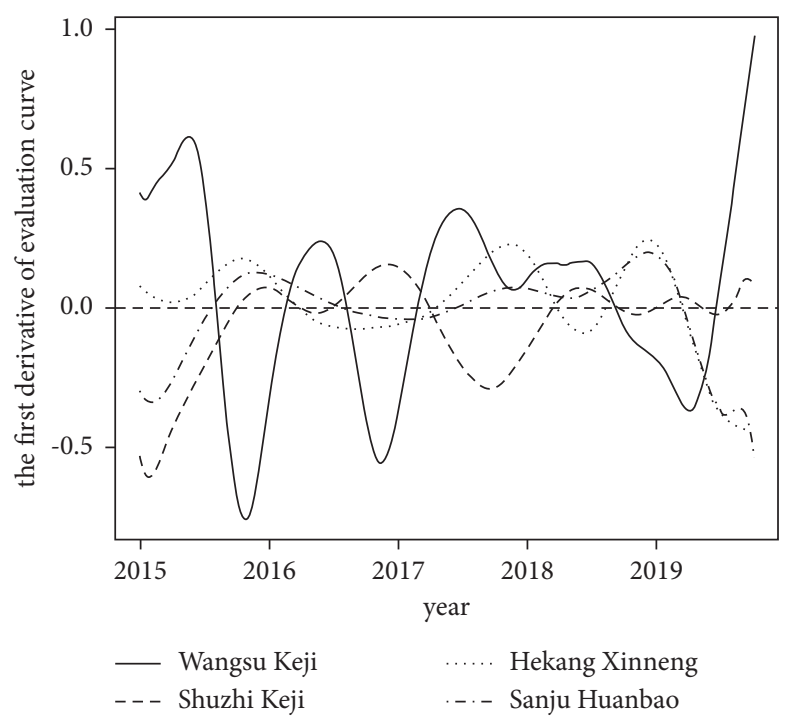

FIgURE 3: Graph of the first derivative of comprehensive evaluation.

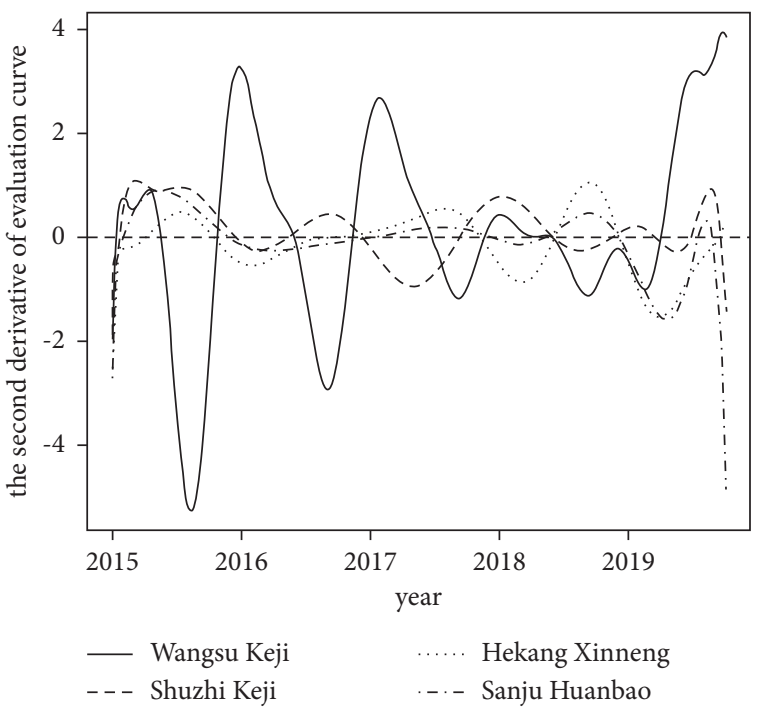

FIGURE 4: Graph of the second derivative of comprehensive evaluation.

overall financial condition can be seen. The rate of growth grows fast and slowly changes to drop. A decline has occurred in the fourth quarter of 2018, with a strong rebound in the third quarter of 2019. The first derivative chart shows that its growth rate greatly outpaces that of other enterprises. The fastest growing curve is the second derivative (acceleration), which hits the peak at the end of 2019. Rapid optimizing development has been preserved by the financial situation of Wangsu Keji in the second half of 2019.

From Figure 2, we find that the evaluation curve of Shuzhi Keji declines significantly in the first three quarters of 2015 , and from the second quarter to the fourth quarter of 2017 , it reaches its lowest point quarter of 2018. Although it started to rise in the fourth quarter in 2015, the fluctuation of speed is between 0 and 0.1 in Figures 3 and 4, with the slowing acceleration and upward trend. From the second quarter of 2018 to the end of the fourth quarter of 2019, although there is an upward trend, speed fluctuation is controlled with a slowing upward trend around 0 .

The dynamic evaluation curve of Hekang Xinneng falls to its lowest point in the first quarter of 2015, followed by a rising strong upward trend in 2015, the second, third, and fourth quarters of 2017, and especially 2018, as shown in Figure 2. As shown in Figures 3 and 4, it has reached a peak of accelerated rising speed in late 2018 and early 2019. There is a relatively flat decline in the second, third, and fourth quarters of 2016 and also a relatively flat decline in the second quarter of 2018, with a sharp downward trend in 2019.

From Figure 2, we can also see that although the overall dynamic evaluation curve of Sanju Environmental Protection is showing an upward trend, there are also two strong decline stages, namely, in the first and second quarters of 2015 and the second, third, and fourth quarters of 2019. 
TABLE 3: Comprehensive dynamic's evaluation value and ranking.

\begin{tabular}{lcccc}
\hline Enterprise & Wangsu Keji & Shuzhi Keji & Hekang Xinneng & Sanju Huanbao \\
\hline Evaluation value & 0.01543127 & 0.01361833 & 0.01512252 & 0.01558047 \\
Ranking & 2 & 4 & 3 & 1 \\
\hline
\end{tabular}

TABLE 4: Evaluation value (EV) and ranking (R) of first-level indicators.

\begin{tabular}{|c|c|c|c|c|c|c|c|c|c|c|c|c|c|}
\hline \multirow{2}{*}{ Enterprise } & \multirow[t]{2}{*}{$\mathrm{R}$} & \multicolumn{2}{|c|}{$\begin{array}{l}\text { Debt paying } \\
\text { ability }\end{array}$} & \multicolumn{2}{|c|}{ Profitability } & \multicolumn{2}{|c|}{$\begin{array}{l}\text { Operating } \\
\text { ability }\end{array}$} & \multicolumn{2}{|c|}{$\begin{array}{l}\text { Growth } \\
\text { ability }\end{array}$} & \multicolumn{2}{|c|}{ Cash flow } & \multicolumn{2}{|c|}{$\begin{array}{c}\text { Innovation } \\
\text { ability }\end{array}$} \\
\hline & & $\mathrm{EV}$ & $\mathrm{R}$ & $\mathrm{EV}$ & $\mathrm{R}$ & $\mathrm{EV}$ & $\mathrm{R}$ & Ev & $\mathrm{R}$ & Ev & $\mathrm{R}$ & Ev & $\mathrm{R}$ \\
\hline Wangsu Keji & 2 & 0.00444 & 4 & 0.00169 & 2 & 0.00380 & 1 & 0.00103 & 4 & 0.00272 & 3 & 0.00275 & 1 \\
\hline Shuzhi Keji & 4 & 0.00447 & 3 & 0.00170 & 1 & 0.00278 & 2 & 0.00121 & 1 & 0.00175 & 2 & 0.00172 & 4 \\
\hline Hekang Xinneng & 3 & 0.00623 & 2 & 0.00141 & 4 & 0.00255 & 4 & 0.00105 & 4 & 0.00157 & 4 & 0.00232 & 2 \\
\hline Sanju Huanbao & 1 & 0.00624 & 1 & 0.00151 & 3 & 0.00274 & 3 & 0.00105 & 3 & 0.00177 & 1 & 0.00227 & 3 \\
\hline
\end{tabular}

However, there is a smaller decline in the third and fourth quarters of 2016 and the first and second quarters of 2017. In Figure 3, it approximates to $\mathrm{M}$ type in the overall trend of the first derivative of the evaluation curve. Most speeds in the intermediate stage are greater than 0 . Especially in Figure 4, the second derivative is greater than that in the third quarter of 2015 and the second quarter to fourth quarter of 2018, while an increasing trend in the first derivative illustrates the rapid development of the enterprise financial conditions during the two stages.

The function data of the comprehensive evaluation function curves have been obtained through the above empirical analysis. If further comprehensive comparative analysis of the financing risks of the four companies is required, the evaluation curve needs to be integrated to obtain the corresponding evaluation results. At the same time, the evaluation results change over different times during the observation period. In practical applications, dynamic analysis tends to pay more attention to recent results, so the weight of the recent period is larger, and the weight of the earlier period is relatively small. Based on the experience of all aspects, the recent data have the greatest impact on the comprehensive evaluation. Therefore, $\alpha=0.1$ is taken in this article by referring to the relevant literature.

The comprehensive dynamic evaluation's values of different enterprises can be obtained by using formula (19) and the result is shown in Table 3:

According to Table 3, the financial risk ranking of each enterprise is

$$
\begin{aligned}
\text { Sanju Huanbao } & >\text { Wangsu Keji }>\text { Hekang Xinneng } \\
& >\text { Shuzhi Keji. }
\end{aligned}
$$

Shuzhi Keji has the largest financial risk, whereas Sanju Huanbao has the smallest financial risk. At the same time, the dynamic comprehensive evaluation value and ranking of the first-level indicators of each enterprise are calculated as shown in Table 4.

The results in Table 4 show that the two enterprises ranks No. 1 and No. 2 point to the first ranking of first-level indicators evaluation values. At the same time, the dispersion degrees of the evaluation value of the six first-level indicators evaluation values are different among the companies. There are differences in the impact on the total value of the dynamic comprehensive evaluation. Therefore, it is necessary to calculate the coefficient of variation of the evaluation value of each first-level index and draw the radar chart, as shown in Figure 5.

In Figure 5, first of all, debt paying ability shows the largest difference in the first-level indicator. Due to the first ranking of first-level evaluation value, the first ranking of the dynamic comprehensive evaluation value is Sanju Huanbao. Secondly, operating ability and innovation ability are the first-level indicators with a large difference. It can be seen that the evaluation value of Wangsu Keji ranks first in these two first-level indicators, leading to the second ranking of the dynamic comprehensive evaluation value. In addition, the first-level indicator (the indicator of debt paying ability and innovation ability) with the prominent coefficient of variation will significantly improve the ranking of the dynamic comprehensive evaluation. In contrast, the first-level indicator with the insignificant coefficient of variation (the indicator of profit and growth ability) has a weak impact on the dynamic comprehensive evaluation results. For example, Shuzhi Keji ranks first in terms of profitability and growth ability, with the rank of third in debt paying ability and fourth in innovation ability. As a result, Shuzhi Keji gets fourth place in the dynamic comprehensive evaluation result. Finally, although Hekang Xinneng does not rank first with the first-level index, it ranks second in two first-level indicators of solvency and innovation ability, the dynamic comprehensive evaluation result of Hekang Xinneng exceeds Shuzhi Keji to rank third.

In order to further analyze the change of the financial risk level, during the observation period from 2015 to 2017 and 2018 to 2019, the above method is used to calculate the dynamic comprehensive evaluation value of each subperiod, as shown in Table 5.

In the first stage, there is a difference in the rank of dynamic comprehensive evaluation value from 2015 to 2017 and the five-year dynamic comprehensive evaluation. In the second stage, the rank of dynamic comprehensive evaluation value during 2018 to 2019 is basically consistent with the results of the five-year dynamic comprehensive evaluation, 


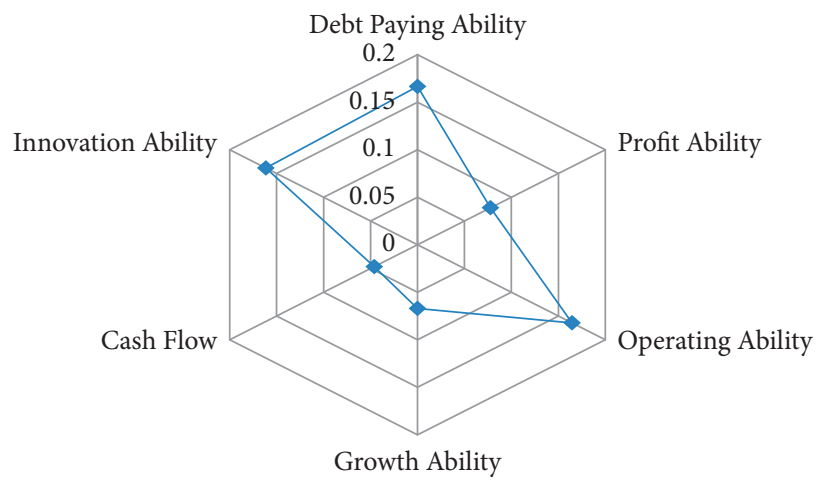

FIgURE 5: Coefficient of the variation radar chart.

TABLE 5: Dynamic comprehensive evaluation value and ranking for two time periods.

\begin{tabular}{|c|c|c|c|c|}
\hline \multirow{2}{*}{ Enterprise } & \multicolumn{2}{|c|}{ From 2015 to 2017} & \multicolumn{2}{|c|}{ From 2018 to 2019} \\
\hline & Evaluation value & Ranking & Evaluation value & Ranking \\
\hline Wangsu Keji & 0.02214174 & 3 & 0.03285694 & 2 \\
\hline Shuzhi Keji & 0.02280446 & 2 & 0.02907365 & 4 \\
\hline Hekang Xinneng & 0.02150332 & 4 & 0.03188928 & 3 \\
\hline Sanju Huanbao & 0.02359825 & 1 & 0.03305203 & 1 \\
\hline
\end{tabular}

which indicates that the development of each enterprise has a great change in the two periods. It can be seen from the ranking results that the dynamic comprehensive evaluation values of Wangsu Keji and Hekang Xinneng have a rising trend, while that of Shuzhi Keji is has a decreasing trend.

\section{Conclusion}

In this article, a new dynamic comprehensive evaluation method is constructed using the functional evaluation method, while the maximum information entropy method describes the dynamic financial risks of an intellectual property pledge financing of listed enterprises. The main conclusions of this article are as follows: first, with the transformation of the quarterly financial observation data of all enterprises into a function curve, the dynamic evaluation curve of each enterprise is obtained, accompanied by the first and second derivatives of the evaluation function curve, to analyze the changing trend of the evaluation curve. At the same time, with the combination of the dynamic evaluation curve with the optimal time weight curve, it is more accurate to calculate the total period value of financing risk. Second, the financial risk of each enterprise is mainly affected by the first-level indicators with outstanding advantages of coefficient of variation, while enterprises with small risk may also have weak influence. The empirical analysis shows that under the guidance of innovative development concepts, the advantages and development characteristics of the enterprise are highlighted to foster strengths and avoid weaknesses, making it necessary to strengthen financial management ability and innovation ability and reduce the risk of enterprise pledge financing. More importantly, the FDA method is applied to analyze the financial risk of enterprise pledge financing dynamically, in favor of objectively evaluating the financial risk of enterprise, which is subject to provide a new evaluation method for the financial risk analysis of high-tech enterprise pledge financing.

\section{Data Availability}

The data used to support the findings of this study are available from the corresponding author upon request.

\section{Conflicts of Interest}

The authors declare that there are no conflicts of interest regarding the publication of this manuscript.

\section{Acknowledgments}

This work was supported by the National Natural Science Foundation of China under Grant nos. 71901222 and 71974204. This work was supported in part by the $\mathrm{Hu}-$ manities and Social Science Foundation of the Ministry of Education of China under Grant no. 21YJC790065, "the Fundamental Research Funds for the Central Universities", and Zhongnan University of Economics and Law under Grant nos. 2722020JCG062 and 2722020JX005.

\section{References}

[1] A. V. Banerjee, B. Timothy, and T. W. Guinnane, "The neighbor's keeper: the design of a credit cooperative with theory and a test," Quarterly Journal of Economics, vol. 109, no. 2, pp. 491-515, 1994.

[2] P. Angelini, R. D. Salvo, and G. Ferri, "Availability and cost for small businesses: customer relationships and credit cooperative," Journal of Banking and Finance, vol. 22, pp. 925-954, 1998.

[3] A. N. Berger and G. F. Udell, "A more complete conceptual framework for SME finance," Journal of Banking and Finance, vol. 30, no. 11, pp. 2945-2966, 2006. 
[4] W. Zhang and X. D. Zhang, "New Financing Mode for small and medium enterprises coordinating the interests among the guarantee institution dominated participants," The Theory and Practice of Finance and Economics, vol. 34, no. 1, pp. 2-6, 2013, in Chinese.

[5] Y. F. Lin and Y. J. Li, "Promoting the growth of medium and small-sized enterprises through the development of medium and small-sized financial institutions," Economic Research Journal, vol. 1, pp. 10-18, 2001, in Chinese.

[6] Z. Xu and C. W. Zou, "Allocation and incentive Mechanism design of loan approval right in banks under the framework of hard and soft information: enlightenment on financing problems of small and medium-sized enterprises," Journal of Financial Research, vol. 8, pp. 1-15, 2010, in Chinese.

[7] X. Xiong, C. Guo, W. Zhang, and Y. J. Zhang, "Loan rate pricing of SME financing based on agent-based computational finance approach," Systems Engineering-Theory \& Practice, vol. 29, no. 12, pp. 9-14, 2009, in Chinese.

[8] X. Xiong, J. M. Tan, W. Zhang, Y. J. Zhang, and Y. Yang, "Study on counter guarantee and proportional guarantee mechanism of SME financing," Soft Science, vol. 25, no. 6, pp. 80-85, 2011, in Chinese.

[9] K. P. Jarboe and I. Ellis, "Intangible assets: innovative financing for innovation," Issues in Science and Technology, vol. 26, no. 2, pp. 75-80, 2010.

[10] J. Crawford and R. Strasser, "Management of infringement risk of intellectual property assets," Intellectual Property and Technology Law Journal, vol. 20, no. 12, pp. 7-10, 2008.

[11] R. Chessy and O. Toivanen, "Is there adverse selection in the credit market?" Venture Capital: An International Journal of Entrepreneurial Finance, vol. 3, no. 3, pp. 215-238, 2001.

[12] J. P. Niinimaki, "Nominal and true cost of loan collateral," Journal of Banking and Finance, vol. 35, no. 10, pp. 2782-2790, 2011.

[13] I. Davies, "Secured financing of intellectual property assets and the reform of English personal property security law," Oxford Journal of Legal Studies, vol. 26, no. 3, pp. 559-583, 2006.

[14] R. Pennington and C. Sanchez, "Negotiating liability allocation terms: risk, indemnity, and intellectual property," Contract Management, vol. 47, pp. 42-53, 2007.

[15] S. Schenkerman, "Avoiding rank reversal in AHP decisionsupport models," European Journal of Operational Research, vol. 74, no. 3, pp. 407-419, 1994.

[16] S. J. Chen and C. L. Hwang, Fuzzy Multiple Attribute Decision Making, Springer, Berlin, Germany, 1992.

[17] T. Ding, J. Yang, H. Wu, W. Yao, C. Tan, and L. Liang, "Research performance evaluation of Chinese university: a non-homogeneous network DEA approach," Journal of Management Science and Engineering, 2020.

[18] A. Liu, T. Liu, J. Mou, and R. Wang, "A supplier evaluation model based on customer demand in block chain tracing anti-counterfeiting platform project management," Journal of Management Science and Engineering, vol. 5, no. 3, pp. 172-194, 2020.

[19] J. O. Ramsay, "When the data are functions," Psychometrika, vol. 47, no. 4, pp. 379-396, 1982.

[20] J. Q. Ramsay and C. J. Dalzell, "Some tools for functional data analysis," Journal of the Royal Statistical Society: Series B, vol. 53, no. 3, pp. 539-572, 1991.

[21] B. W. Silverman, "Smoothed functional principal components analysis by choice of norm," Annals of Statistics, vol. 24, no. 1, pp. 1-24, 1996.
[22] Z. Lu, "Nonparametric functional data analysis: theory and practice," Technometrics, vol. 49, no. 2, pp. 225-226, 2007.

[23] A. Delagle and P. Hall, "Defining probability density for a distribution of random function," Annals of Statistics, vol. 38, no. 2, pp. 1171-1193, 2010.

[24] A. Petersen and H. G. Muller, "Functional data analysis for density functions by transformation to a Hilbert space," Annals of Statistics, vol. 44, no. 1, pp. 183-218, 2016.

[25] P. Kokoszka and M. Reimherr, Introduction to Functional Data Analysis, Chapman Hall/CRC, FL, USA, 2017.

[26] T. Hastie, A. Buja, and R. Tibshirani, "Penalized disciminant analysis," Annals of Statistics, vol. 23, no. 1, pp. 73-102, 1995.

[27] G. M. James, "Generalized linear models with functional predictors," Journal of the Royal Statistical Society: Series B, vol. 64, pp. 422-432, 2002.

[28] J. O. Ramsay, G. Hooker, D. Campbell, and J. Gao, "Parameter estimation for differential equations: a generalized smoothing approach," Journal of the Royal Statistical Society: Series B, vol. 16, pp. 741-796, 2007.

[29] F. Yao, H. G. Muller, and J. L. Wang, "Functional data analysis for longitudinal data," Annals of Statistics, vol. 23, pp. 2973-2903, 2005.

[30] N. Serban and L. Wasserman, "Cats: clustering after transformation and smoothing," Journal of the American Statistical Association, vol. 100, no. 1, pp. 990-999, 2005.

[31] J. Zhang, "Risk management on intellectual property pledge financing by small and medium $S$ \& T enterprises: from the aspect of bank," Scientific Management Research, vol. 31, no. 2, pp. 98-101, 2013.

[32] H. Li, Z. Yuan, and S. Li, "Innovative enterprises intellectual property pledge loan risk assessment," Studies in Science of Science, vol. 35, no. 8, pp. 1253-1263, 2017.

[33] W. H. Su, L. R. Sun, and F. Cui, "One comprehensive evaluation method study based on functional data," Statistical Research, vol. 2, pp. 88-94, 2013, in Chinese.

[34] M. Y. Yan, "The evaluation of quality of life a method based on functional features of data," Journal of Statistics and Information, vol. 2, pp. 13-17, 2007, in Chinese.

[35] Y. J. Guo, Y. Yao, and P. T. Yi, "A dynamic comprehensive evaluation method and its application," Systems Engineering-Theory \& Practice, vol. 10, pp. 154-158, 2007, in Chinese. 